\title{
VIIRS ON-ORBIT CALIBRATION AND PERFORMANCE UPDATE
}

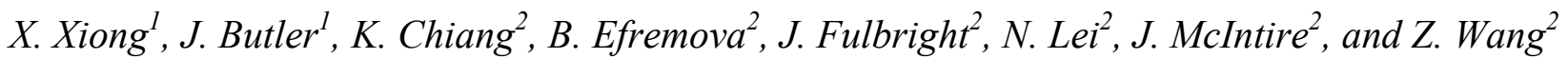 \\ ${ }^{1}$ Sciences and Exploration Directorate, NASA/GSFC, Greenbelt, MD 20771, USA \\ ${ }^{2}$ Sigma Space Corporation, 4600 Forbes Boulevard, Lanham, MD 20706, USA
}

\begin{abstract}
The S-NPP VIIRS was launched on October 28, 2011 and activated on November 8 , and then went through a series of intensive functional tests in order to establish the sensor's baseline characteristics and initial on-orbit performance. With the exception of large optical degradation in the NIR and SWIR spectral regions that is due to pre-launch mirror coating contamination, both the VIIRS instrument and its on-board calibrators continue to operate and function normally. With continuous dedicated effort, it is expected that most of the sensor calibration parameters will continue to meet their design requirements and that high quality data products will be continuously generated and used by the operational as well as research community.
\end{abstract}

Index Terms - VIIRS, S-NPP, SDR, Calibration, VCST

\section{INTRODUCTION}

The first Visible Infrared Imaging Radiometer Suite (VIIRS) has successfully operated for more than two years since its launch on-board the Suomi-National Polar-orbiting Partnership (S-NPP) spacecraft. The VIIRS has 22 spectral bands: 16 moderate resolution bands (M1-M16; $750 \mathrm{~m}$ at nadir) that are primarily used for radiometric observations, 5 imagery bands (I1-I5; $375 \mathrm{~m}$ at nadir), and 1 day/night band (DNB) of 750 m nadir resolution. M1-M11, I1-I3, and DNB are the reflective solar bands (RSB) covering wavelengths from 0.41 to $2.3 \mu \mathrm{m}$. M12-M16 and I4-I5 are the thermal emissive bands (TEB) covering wavelengths from 3.7 to 12 $\mu \mathrm{m} .7$ of the 22 spectral bands; M1-M5, M7, and M13 are the dual gain bands, enabling observations to be made over a large dynamic range. VIIRS on-orbit calibration is provided by a set of on-board calibrators (OBC) designed with a strong MODIS heritage, which include a solar diffuser (SD), a solar diffuser stability monitor (SDSM), and a blackbody (BB) as shown in Figure 1. Since launch, the NASA VIIRS Characterization Support Team (VCST), in support of the S-NPP mission operation team (MOT), has scheduled and carried out a broad range of VIIRS radiometric and geometric calibration activities, which have led to the generation of consistent calibration look-up tables (LUT) for improved Sensor Data Records (SDR) production. This effort has significantly contributed to the VIIRS SDR as well as the Environmental Data Records (EDR) quality assessment and validation. This paper provides an overview of VIIRS calibration and characterization activities performed since its launch and an update to its on-orbit performance. Results derived from the sensor $\mathrm{OBC}$ and lunar observations are illustrated. Challenging issues and potential improvements for VIIRS SDR calibration and data quality are also briefly discussed.

\section{CALIBRATION ACTIVITIES}

The VIIRS RSB calibration is referenced to the bidirectional reflectance factor (BRF) of its on-board SD. It has a fixed SD attenuation screen (SAS) but does not have a SD aperture door like MODIS. As a result, the SD calibration data is available each orbit. The SDSM, designed to track on-orbit changes of the SD BRF, was mostly operated on a daily basis for two and a half years and then changed to 3 times weekly starting from May 16, 2014. In addition to SD and SDSM calibrations, near-monthly lunar observations are scheduled and used to provide independent monitoring of RSB calibration stability. Spacecraft roll maneuvers are often executed during scheduled lunar calibration events in order to keep the observed lunar phase angles nearly the same. To date, VIIRS has performed a total of 20 scheduled lunar observations.

The TEB calibration is performed using the $\mathrm{OBC} \mathrm{BB}$, which is nominally controlled at $292.5 \mathrm{~K}$ and goes through, on a quarterly basis, a warm-up and cool-down (WUCD) cycle with its temperatures varying between instrument ambient (about $267 \mathrm{~K}$ ) and $315 \mathrm{~K}$. Since launch, VIIRS has performed 9 BB WUCD cycles. Space view (SV) 
observations are made on a scan-by-scan basis and applied for sensor background correction. In addition to roll maneuvers, VIIRS has performed yaw and pitch maneuvers during its intensive calibration and validation (ICV) phase. Results derived from yaw maneuver data sets have led to improvements of key calibration parameters, such as the SDSM Sun view screen transmission, and the combination of SD BRF and SAS transmission. Pitch maneuver data has been successfully used to validate the TEB response versus scan-angle (RVS) of the half angle mirror (HAM).
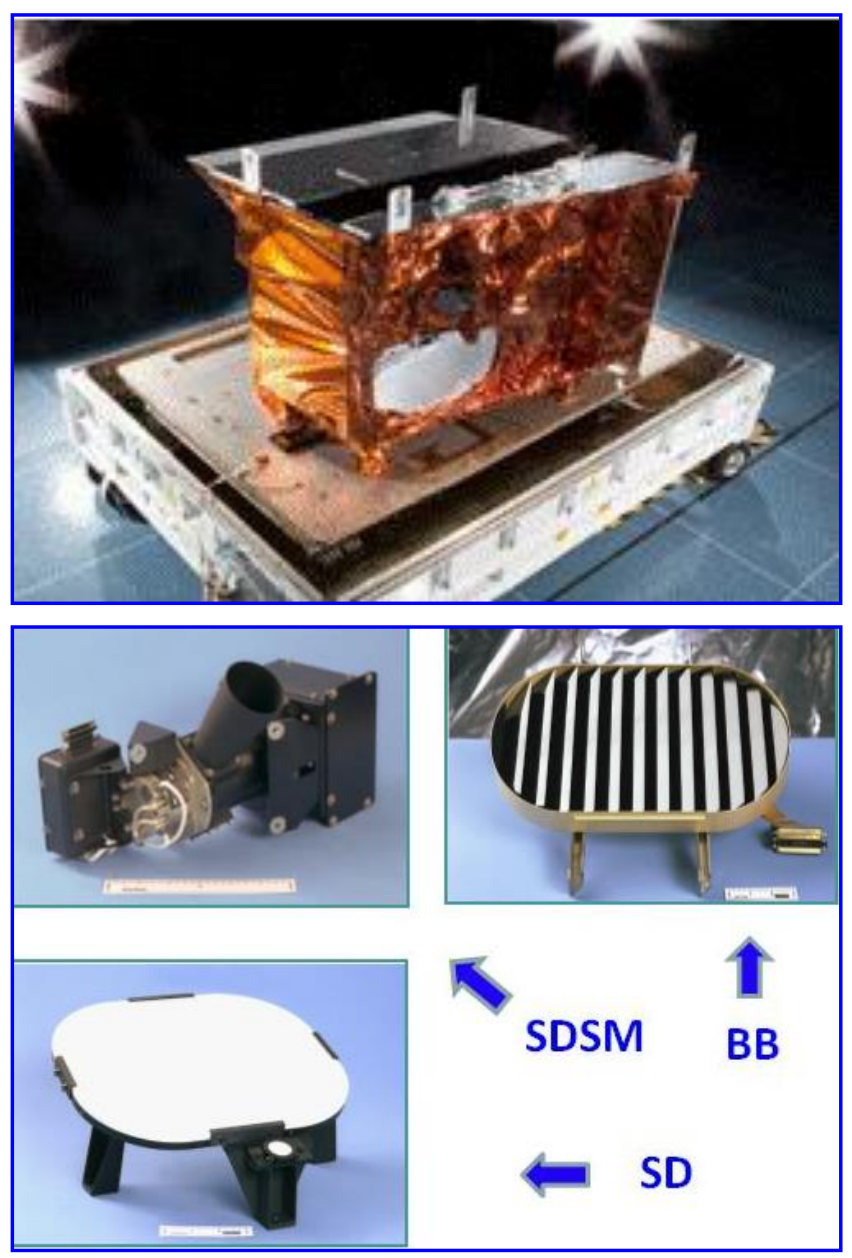

Fig. 1 VIIRS instrument mainframe (top) and its on-board calibrators (bottom): solar diffuser (SD), solar diffuser stability monitor (SDSM), and blackbody (BB).

\section{ON-ORBIT PERFORMANCE}

Details of VIIRS on-orbit calibration methodologies and initial on-orbit performance can be found in a number of references [1-3]. For the RSB calibration, a scaling factor (F-factor), inversely proportional to the detector gain, is derived from SD and SDSM observations. Figure 2 shows the normalized $1 / \mathrm{F}$-factors, or the normalized radiometric gains, (solid lines) for VIIRS VIS/NIR bands I1, I2, and M4-M7 and for SWIR bands I3 and M8-M11. The large degradation observed in some of the NIR and SWIR responses (e.g. I2 and M6-M9) is due to coating contamination on the mirrors of the rotating telescope assembly (RTA). For comparison purposes, the normalized radiometric gains derived from lunar observations are also illustrated in Figure 2 (symbols). In general, the F-factors from SD calibration agree well with that derived from lunar calibration. Small but noticeable differences between SD and lunar calibration also exist in their normalized response trending (on the order of $0.5-1.0 \%$ peak-to-peak). Future improvements of SD calibration and lunar model references are being considered to help understand and resolve these differences.
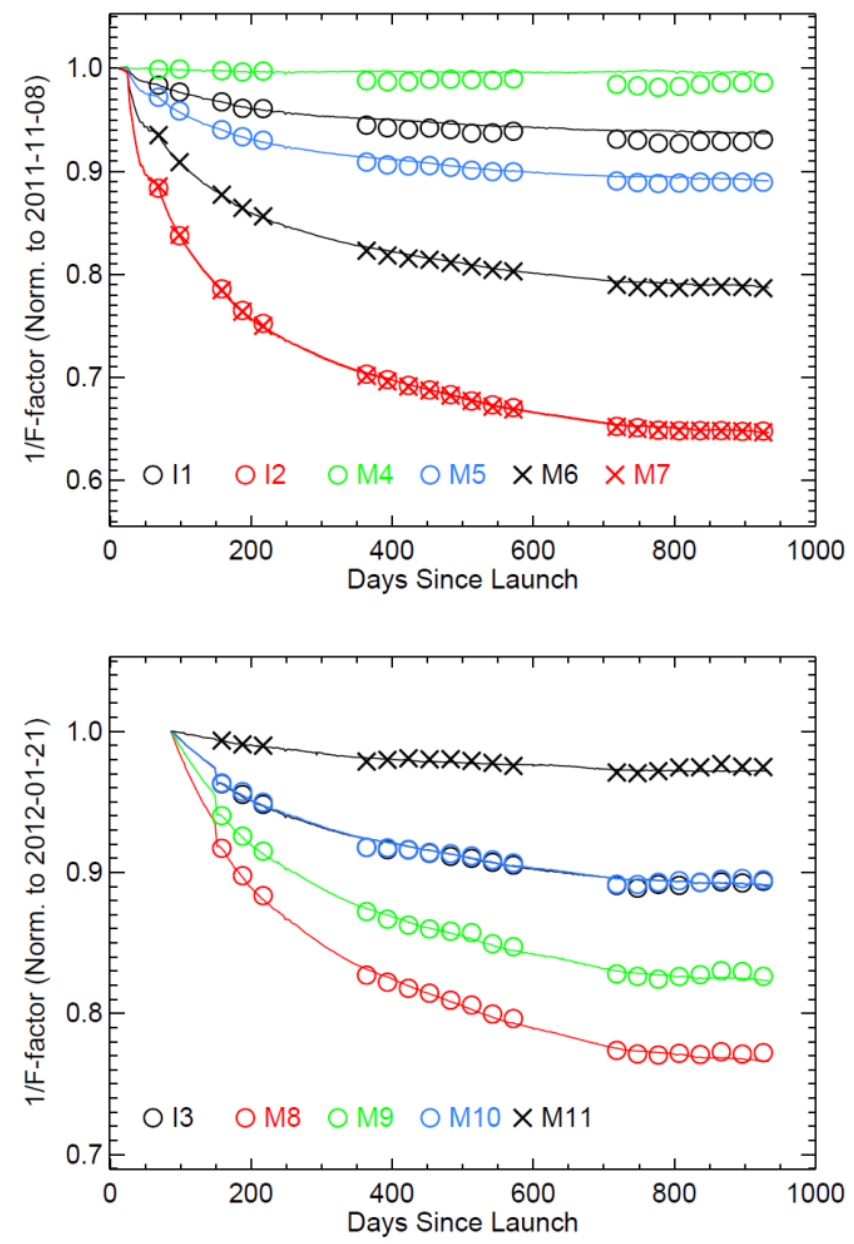

Fig. 2 Normalized radiometic gains (1/F-factor) of VIIRS VIS/NIR bands (top) and SWIR bands (bottom): band averaged, high gain state, HAM side A. Solid lines are from SD/SDSM calibration and symbols from lunar calibration. 
The F-factors are derived accounting for the SD BRF degradation, which is tracked by the SDSM. The SDSM has 8 individual detectors, centered at wavelengths between 0.41 and $0.93 \mu \mathrm{m}$. Its on-orbit performance is illustrated in Figure 3 via its normalized Sun-view responses (top). Detector $8(0.93 \mu \mathrm{m})$ has experienced the largest change at $32 \%$, followed by detector $7(0.86 \mu \mathrm{m})$ at $17 \%$. Changes in the remaining detectors' responses are generally within 5\%. Also illustrated in Figure 3 are the SD degradation trends at different detector wavelengths (bottom), where the larger degradation occurs at shorter wavelength. VIIRS SDSM and SD performance is very similar to MODIS [4].
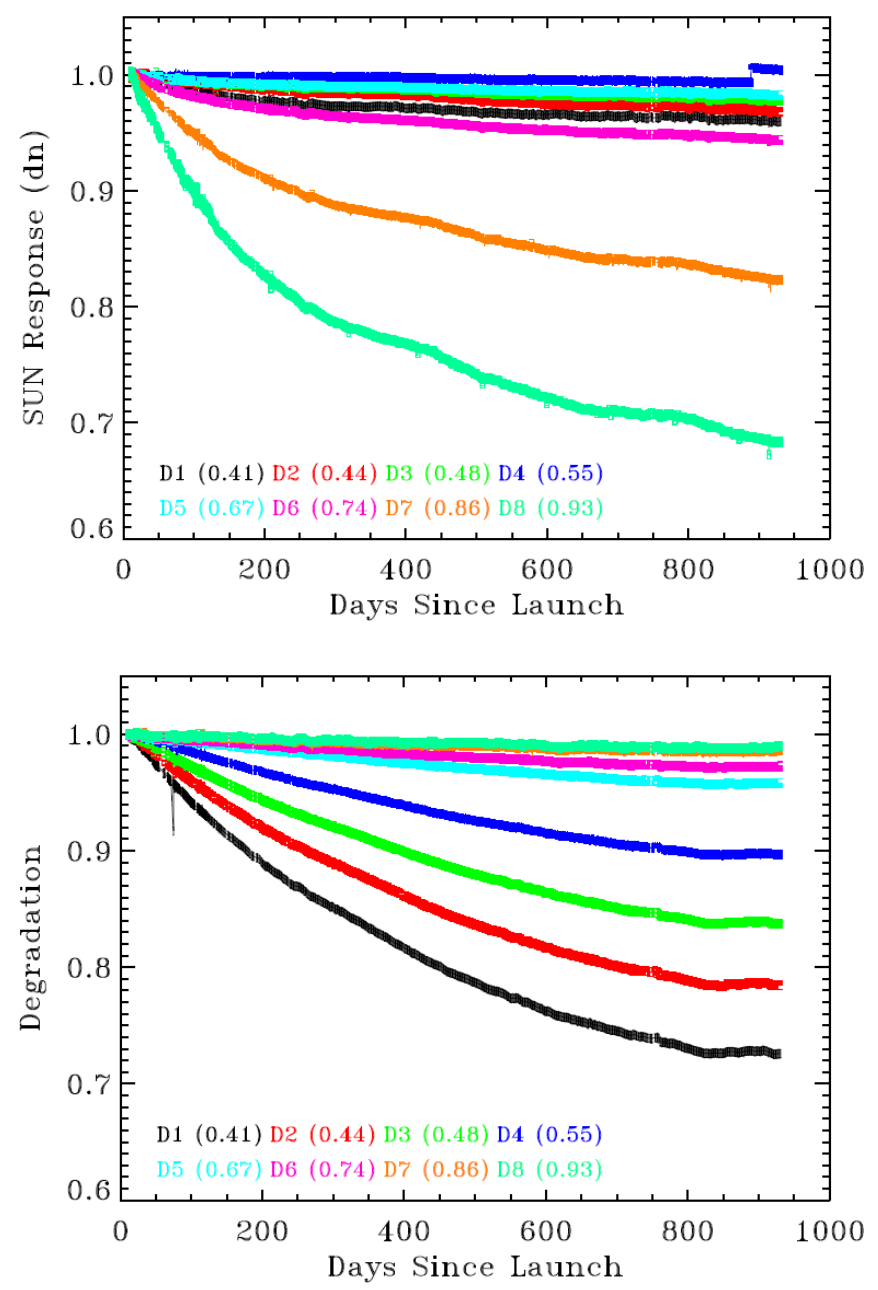

Fig. 3 VIIRS SDSM Sun view response trending (top) and SD degradation trending (bottom) at different wavelengths $(0.41-0.93$ $\mu \mathrm{m})$.

It was discovered in early 2014 that the solar vector in the SNPP data was not accurate since launch, which was caused by the misuse of two different Earth centered inertial reference frames in the common geolocation library at the NOAA Interface Data Processing Segment (IDPS) system. Because of this, extensive impact assessments have been made and correction strategies examined. It is expected that this error could have a small but noticeable impact on some radiometric calibration parameters; e.g. $0.2 \%$ on $\mathrm{H}$-factors and $0.2 \%$ for the SWIR F-factors. Figures 2 and 3 are derived using corrected mission solar vectors generated by VCST.

The calibration impact due to wavelength dependent degradation shown in Figure 2 has been continuously and extensively studied by the VCST via a mirror degradation model [5]. In addition to development of modulated relative spectral responses (RSR), this model is used to predict future sensor performance in terms of signal to noise ratios (SNR). Unlike RSB, changes in TEB detector responses have been very small. Data from BB WUCD operations are used to validate the TEB detector offset and nonlinear calibration coefficients and to characterize the detector noise-equivalent temperature difference (NEdT) over a range of temperatures. The VIIRS RSB SNR and TEB NEdT performance, from launch to present, is demonstrated in Figure 4 and summarized in Table 1. On-orbit results are normalized to their corresponding specified requirements for SNR. SNR/SNR spec $>1$ or NEdT $<\mathrm{NEdT}_{\text {spec }}$ indicate that sensor performance meets the requirements.

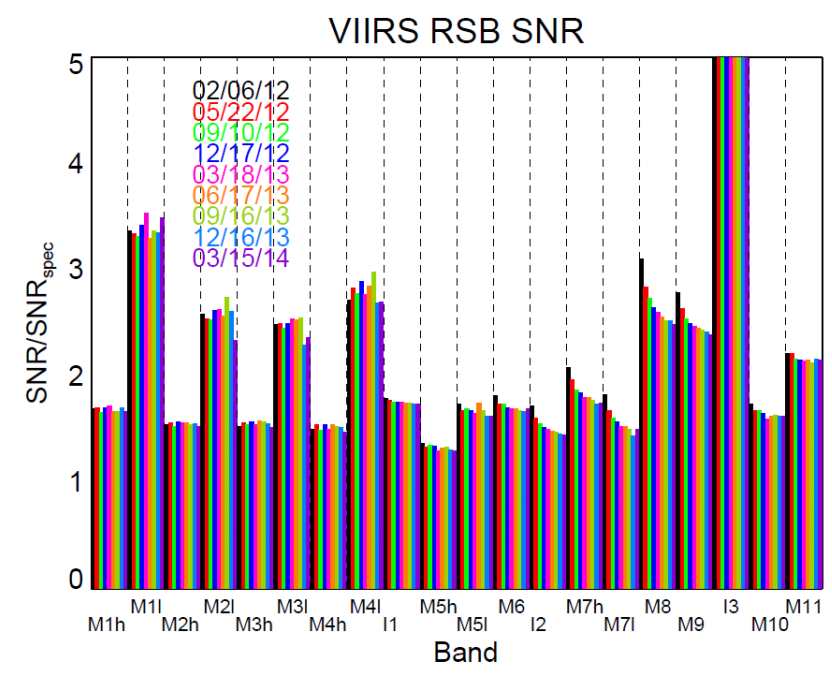

Fig. 4 VIIRS RSB signal to noise ratios (SNR). The SNR results are nomarlized to their corresponding specified values.

Since October 2012, a number of consistently processed and improved LUTs have been produced by VCST to support the SDR reprocessing effort by the NASA Land Product 
Evaluation and Analysis Tools Element (PEATE). Improvements include the use of time dependent modulated RSR, due to RTA mirrors degradation, for the RSB starting from mission beginning, smooth functions applied to the predicted F-factor LUT, the DNB stray light correction LUT derived from the Earth view data under new Moon, and the revised transmission functions of the SD and SDSM screens using the corrected solar vector. Currently, no SD degradation is applied to SWIR bands calibration as the onboard SDSM only covers the wavelengths in the VIS and NIR regions. Although the current impact could be small, future improvements are being considered by combining onorbit lunar calibration.

\begin{tabular}{|c|c|c|c|c|c|c|c|c|c|c|c|}
\hline \multicolumn{3}{|c|}{ VIIRS TEB Specification } & \multicolumn{9}{|c|}{ On-orbit NEdT $[K]$ at $T_{T Y P}$ Trending } \\
\hline Band & $\begin{array}{l}\mathbf{T}_{\mathrm{TrP}} \\
\text { [K] }\end{array}$ & $\begin{array}{l}\text { NEdT Req. } \\
\text { @ } \mathrm{T}_{\mathrm{TYP}}[\mathrm{K}]\end{array}$ & $\begin{array}{c}\text { Feb } \\
2012\end{array}$ & $\begin{array}{l}\text { May } \\
2012\end{array}$ & $\begin{array}{c}\text { Sep } \\
2012\end{array}$ & $\begin{array}{r}\text { Dec } \\
2012\end{array}$ & $\begin{array}{l}\text { Mar } \\
2013\end{array}$ & $\begin{array}{c}\text { Jun } \\
2013\end{array}$ & $\begin{array}{c}\text { Sep } \\
2013\end{array}$ & $\begin{array}{c}\text { Dec } \\
2013\end{array}$ & $\begin{array}{l}\text { Mar } \\
2014\end{array}$ \\
\hline 14 & 270 & 2.5 & 0.4 & 0.4 & 0.4 & 0.4 & 0.4 & 0.4 & 0.4 & 0.4 & 0.4 \\
\hline 15 & 210 & 1.5 & 0.4 & 0.4 & 0.4 & 0.4 & 0.4 & 0.4 & 0.4 & 0.4 & 0.4 \\
\hline M12 & 270 & 0.396 & 0.13 & 0.13 & 0.13 & 0.11 & 0.12 & 0.12 & 0.12 & 0.12 & 0.12 \\
\hline M13 & 300 & 0.107 & 0.04 & 0.04 & 0.04 & 0.04 & 0.04 & 0.04 & 0.04 & 0.04 & 0.04 \\
\hline M14 & 270 & 0.091 & 0.05 & 0.06 & 0.06 & 0.06 & 0.06 & 0.06 & 0.06 & 0.06 & 0.06 \\
\hline M15 & 300 & 0.07 & 0.03 & 0.03 & 0.03 & 0.03 & 0.03 & 0.03 & 0.03 & 0.03 & 0.03 \\
\hline M16 & 300 & 0.072 & 0.03 & 0.03 & 0.03 & 0.03 & 0.03 & 0.03 & 0.03 & 0.03 & 0.03 \\
\hline
\end{tabular}

Table. 1 VIIRS TEB noise-equivalent temperature difference (NEdT). The $\mathrm{NEdT}_{\text {spec }}$ values are specified at typical Earth scene radiance or brightness temperature.

\section{SUMMARY}

The NASA VCST has been active in the tracking and improvement of the performance of S-NPP VIIRS since launch. For the RSB, VCST has provided, among others, improved transmission screens for the SD calibration, RSR degradation modeling, a correction for the solar vector error, and lunar calibration support. For the TEB, the contribution from the VCST includes complete WUCD data analysis, BB thermistor modeling, and NEdT trending. These activities and the creation of consistent LUTs for SDR reprocessing are the result of a commitment to the continual improvement of VIIRS data products for the at-large science and user community. Since launch, the overall performance of the sensor and its $\mathrm{OBC}$ has been excellent.

\section{ACKNOWLEDGEMENTS}

Authors would like to acknowledge the technical assistance and contributions made by other VCST and VIIRS SDR Team members.

\section{REFERENCES}

[1] Xiong, X., H. Oudrari, K. Chiang, J. McIntire, J. Fulbright, N. Lei, J. Sun, B. Efremova, Z. Wang, and J. Butler, "VIIRS On-Orbit Calibration Activities and Performance," IGARSS, 2013.

[2] Xiong X., J. Butler, K. Chiang, B. Efremova, J. Fulbright, N. Lei, J. McIntire, H. Oudrari, J. Sun, Z. Wang, and A. Wu, "VIIRS On-orbit Calibration Methodology and Performance," (in press JGR 2014).

[3] Cao, C., X. Xiong, F. De Luccia, R. Wolfe, and F. Weng, "Early On-orbit Performance of the Visible Infrared Imaging Radiometer Suite (VIIRS) onboard the Suomi National Polar-orbiting Partnership (SNPP) Satellite," (in press IEEE TGRS).

[4] Xiong, X., J. Fulbright, A. Angal, J. Sun, and Z. Wang, "Comparison of MODIS and VIIRS Solar Diffuser Stability Monitor Performance", Proc. SPIE 8528, Earth Observing Missions and Sensors: Development, Implementation and Characterization II, 852809, 2012.

[5] Lei, N., B. Guenther, Z. Wang, and X. Xiong, "Modeling SNPP VIIRS reflective solar bands optical throughput degradation and its impacts on the relative spectral response," Proc. SPIE 8866, Earth Observing Systems XVIII, 88661H, 2013. 